\title{
A Rare Case of Brain Abscesses Caused by Acremonium Species
}

\author{
Armeena Anis ${ }^{1}$, Fnu Sameeullah ${ }^{2}$, Jamil M. Bhatti ${ }^{3,4}$
}

1. Medicine, Dr. Ziauddin Hospital, Karachi, PAK 2. Internal Medicine, Carney Hospital, Boston, USA 3. Infectious Diseases, The Cancer Foundation Hospital, Karachi, PAK 4. Infectious Diseases, Dr. Ziauddin Hospital, Karachi, PAK

Corresponding author: Armeena Anis, armeena.anis@gmail.com

\begin{abstract}
Acremonium species are saprophytic fungi that are rarely pathogenic in humans. According to several reports, Acremonium species can cause various diseases, ranging from superficial infections after traumatic inoculation in immunocompetent individuals to invasive infections in the immunocompromised. To the best of our knowledge, this is the first case report of brain abscess in an 18-year-old male caused by Acremonium species in Pakistan. A combination of intravenous amphotericin B and oral voriconazole was administered to the patient, which resulted in marked clinical improvement. However, the recurrence of fungi was observed after three months of completion of the antifungal course. The purpose of this report is to alert clinicians regarding this pathogen and its ability to cause systemic disease.
\end{abstract}

Categories: Internal Medicine, Infectious Disease, Neurosurgery

Keywords: acremonium, voriconazole, amphotericin, recurrence, brain abscess, fungal abscess

\section{Introduction}

The genus Acremonium, previously known as Cephalosporium, consists of approximately 150 species of hyaline filamentous fungi. Most of these species are environmental saprophytes that are primarily isolated from soil and decaying plant material [1]. While fungi belonging to the genus Acremonium rarely infect humans; however, when they do, they can infect both immunocompetent and immunocompromised individuals [2]. Several cases of localized and systemic infections involving multiple organs, including the brain, have been reported to be caused by these fungi [3]. Here, we report an unusual case of brain abscesses caused by the Acremonium species in Pakistan.

\section{Case Presentation}

An otherwise healthy 18-year-old male experienced a near-drowning incident in fresh water in June 2020. He was taken to a local hospital after being rescued and resuscitated. He was then treated in the intensive care unit for aspiration pneumonia and was put on ventilator support. Four days later, as the patient's condition improved, he was extubated with a Glasgow Coma Scale score of 15 and oxygen $\left(\mathrm{O}_{2}\right)$ saturation of

Review began 03/29/2021 Review ended 04/06/2021 Published 04/10/2021

\section{○ Copyright 2021}

Anis et al. This is an open access article distributed under the terms of the Creative Commons Attribution License CC-BY 4.0., which permits unrestricted use, distribution, and reproduction in any medium, provided the original author and source are credited.
99\% on room air. The patient was discharged after two more days. After a week, he presented to Dr. Ziauddin Hospital with a temperature of $101^{\circ} \mathrm{F}$ and a one-day history of shortness of breath at rest. During his physical examination, he appeared to be conscious and restless and was found to have tachypnea, temperature of $102^{\circ} \mathrm{F}$, pulse of 108 beats/min, respiratory rate of 26 breaths/min, a blood pressure (BP) of $115 / 75 \mathrm{mmHg}$, and $\mathrm{O}_{2}$ saturation of $90 \%$ on room air. His chest examination revealed harsh vesicular breathing. The rest of the clinical examination was unremarkable.

On admission, laboratory investigations revealed a high total leukocyte count of $20.0 \times 10^{9} / 1$, procalcitonin level of $19.6 \mathrm{ng} / \mathrm{ml}$, erythrocyte sedimentation rate of $>100 \mathrm{~mm} / \mathrm{h}$, and D-dimer level of 3,144 ng/ml fibrinogen equivalent units (FEU), in addition to a markedly elevated C-reactive protein level of $440.05 \mathrm{mg} / \mathrm{l}$ (Table 1). 


\section{Cureus}

\begin{tabular}{|c|c|c|}
\hline Variables & Normal Range & Value on Admission \\
\hline $\mathrm{Hb}(\mathrm{g} / \mathrm{dl})$ & $13.0-17.0$ & 12.3 \\
\hline HCT (\%) & $40-50$ & 36 \\
\hline $\operatorname{TLC}\left(\times 10^{9} / /\right)$ & $11.5-14.5$ & 20.0 \\
\hline Platelets $\left(\times 10^{9} / \mathrm{I}\right)$ & $150-440$ & 798 \\
\hline $\operatorname{ESR}(\mathrm{mm} / \mathrm{h})$ & $0-20$ & $>100$ \\
\hline Sodium (mEq/l) & $136-145$ & 137 \\
\hline Potassium (mEq/l) & $3.5-5.1$ & 2.9 \\
\hline Chloride (mEq/l) & 98-107 & 102 \\
\hline Bicarbonate (mEq/l) & 23-29 & 23 \\
\hline Urea (mg/dl) & 17-49 & 25 \\
\hline Creatinine (mg/dl) & $0.9-1.3$ & 0.74 \\
\hline C-reactive protein (mg/l) & $<5.0$ & 440.05 \\
\hline Procalcitonin (ng/ml) & $<0.046$ & 19.6 \\
\hline D-dimer (ng/ml FEU) & $55-1,550$ & 3,144 \\
\hline Albumin (g/dl) & $3.50-5.20$ & 3.12 \\
\hline Total bilirubin (mg/dl) & $0-2$ & 0.45 \\
\hline Direct bilirubin (mg/dl) & $<0.2$ & 0.21 \\
\hline AST (U/l) & $<35$ & 23 \\
\hline ALT (U/I) & $<45$ & 27 \\
\hline GGT (IU/I) & $<55$ & 30 \\
\hline Alkaline phosphatase (U/l) & $53-128$ & 55 \\
\hline
\end{tabular}

\section{TABLE 1: Baseline laboratory values on the day of admission}

$\mathrm{Hb}$, hemoglobin; HCT, hematocrit; TLC, total leukocyte count; ESR, erythrocyte sedimentation rate; AST, aspartate aminotransferase; ALT, alanine aminotransferase; GGT, gamma-glutamyl transferase

The chest X-ray revealed bilateral patchy infiltrates. Although the patient's blood culture was sterile, his sputum culture revealed two micro-organisms, namely Enterobacter species and Pseudomonas aeruginosa (Table 2). 


\section{Cureus}

\begin{tabular}{|l|c|c|}
\hline Antibiotics & Micro-organism 1 & Micro-organism 2 \\
\hline Amikacin & R & S \\
Aztreonam & R & \\
Cefoperazone/sulbactam & R & \\
Cefixime & R & S \\
Ceftazidime & R & \\
Ceftriaxone & R & $\mathrm{S}$ \\
Colistin & S & \\
Cotrimoxazole & R & $\mathrm{S}$ \\
Gentamicin & R & $\mathrm{S}$ \\
Imipenem & & $\mathrm{S}$ \\
Meropenem & $\mathrm{R}$ & $\mathrm{S}$ \\
Ofloxacin & $\mathrm{R}$ & $\mathrm{S}$ \\
Ciprofloxacin & $\mathrm{R}$ & $\mathrm{S}$ \\
Polymyxins & $\mathrm{S}$ & $\mathrm{S}$ \\
Tazobactam/piperacillin & $\mathrm{R}$ & \\
\hline
\end{tabular}

TABLE 2: Sputum culture and sensitivity report revealing the presence of Enterobacter species (micro-organism 1) and Pseudomonas aeruginosa (micro-organism 2)

$\mathrm{R}$, resistant; S, sensitive

Treatment with inhaled and intravenous colistin was initiated. However, despite the micro-organisms being sensitive to colistin, no clinical improvement was noted, and the patient remained febrile. Blood culture repeated on the 12th day of admission revealed the presence of Acinetobacter species sensitive to colistin. Hence, the administration of colistin was continued. On the 15 th day of admission, the patient became drowsy and lethargic and developed persistent pyrexia of $104^{\circ} \mathrm{F}$. Therefore, magnetic resonance imaging (MRI) of the head was performed and revealed multiple brain abscesses. The largest one was present in the right frontal lobe and was associated with vasogenic edema (Figures $1 A, 1 B, 2 A, 2 B$ ). 


\section{Cureus}

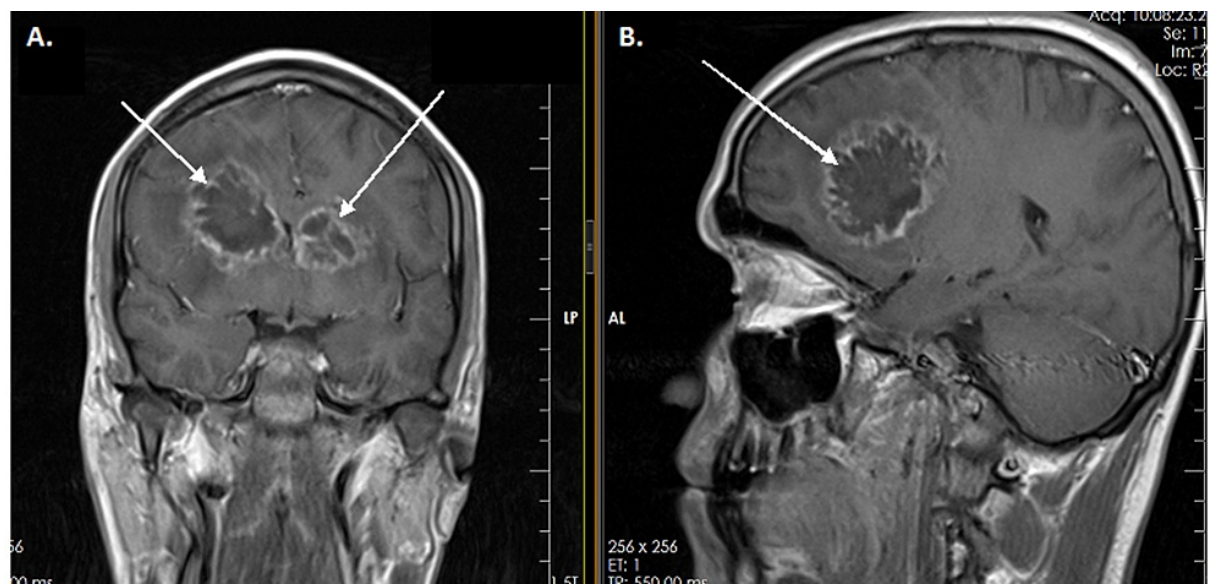

FIGURE 1: Magnetic resonance images of the brain (without contrast) showing arrows pointing to multifocal, lobulated, ring-enhancing lesions in both cerebral hemispheres surrounded with vasogenic edema (A, B)
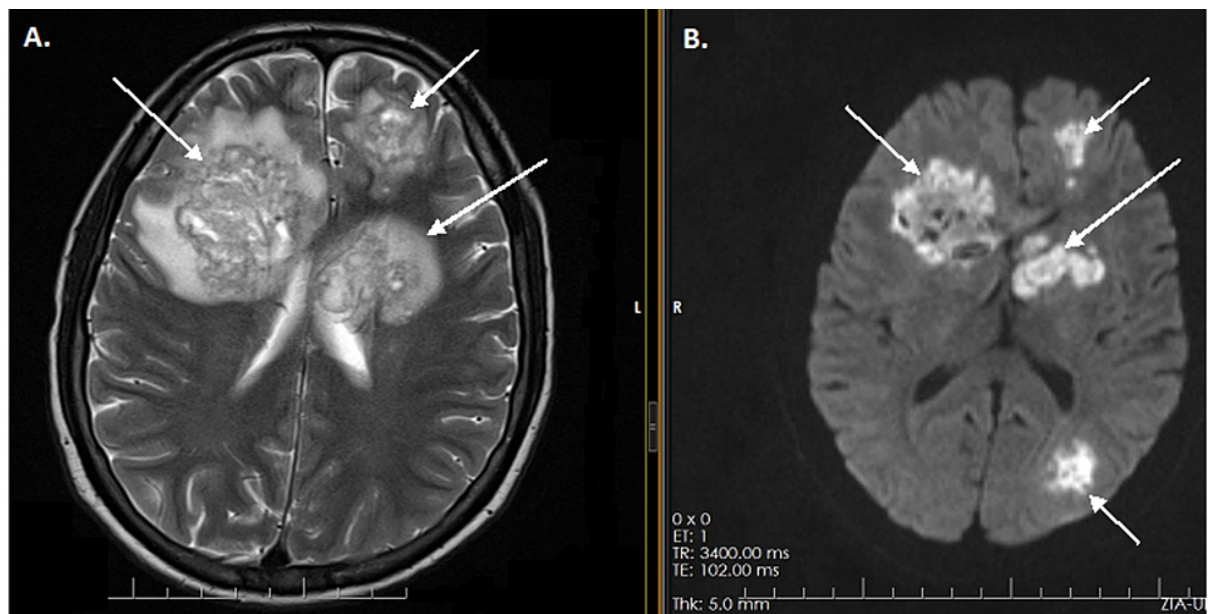

FIGURE 2: Diffusion-weighted magnetic resonance images of the brain with arrows pointing out multiple lesions with restricted diffusion in both cerebral hemispheres, including the basal ganglia, left thalamus, left cerebral peduncle, and right frontal lobe, surrounded with vasogenic edema (A, B)

Consequently, craniotomy and drainage of purulent material from the right frontal abscess were performed. Moderate growth of Acremonium species was noted in the pus extracted during the surgery. Intravenous antifungal therapy with amphotericin B (30 mg once a day) was initiated. However, the patient still remained febrile and drowsy. The antifungal regimen was therefore modified, and voriconazole injection (6 mg/kg twice a day for the first $24 \mathrm{~h}$ and $4 \mathrm{mg} / \mathrm{kg}$ twice a day for the next two days) was added. This was later switched to oral voriconazole (200 $\mathrm{mg}$ twice a day). After administering voriconazole, the patient became afebrile and his neurological status improved. A post-craniotomy computed tomography (CT) scan of the brain revealed partial evacuation of the abscess in the right frontal lobe and multifocal hypodense lesions in both cerebral hemispheres. Recent post-craniotomy changes were noted in the right frontal region, with small punctate hemorrhagic foci being detected in the surgical bed of the right frontal lobe (Figure 3). 


\section{Cureus}

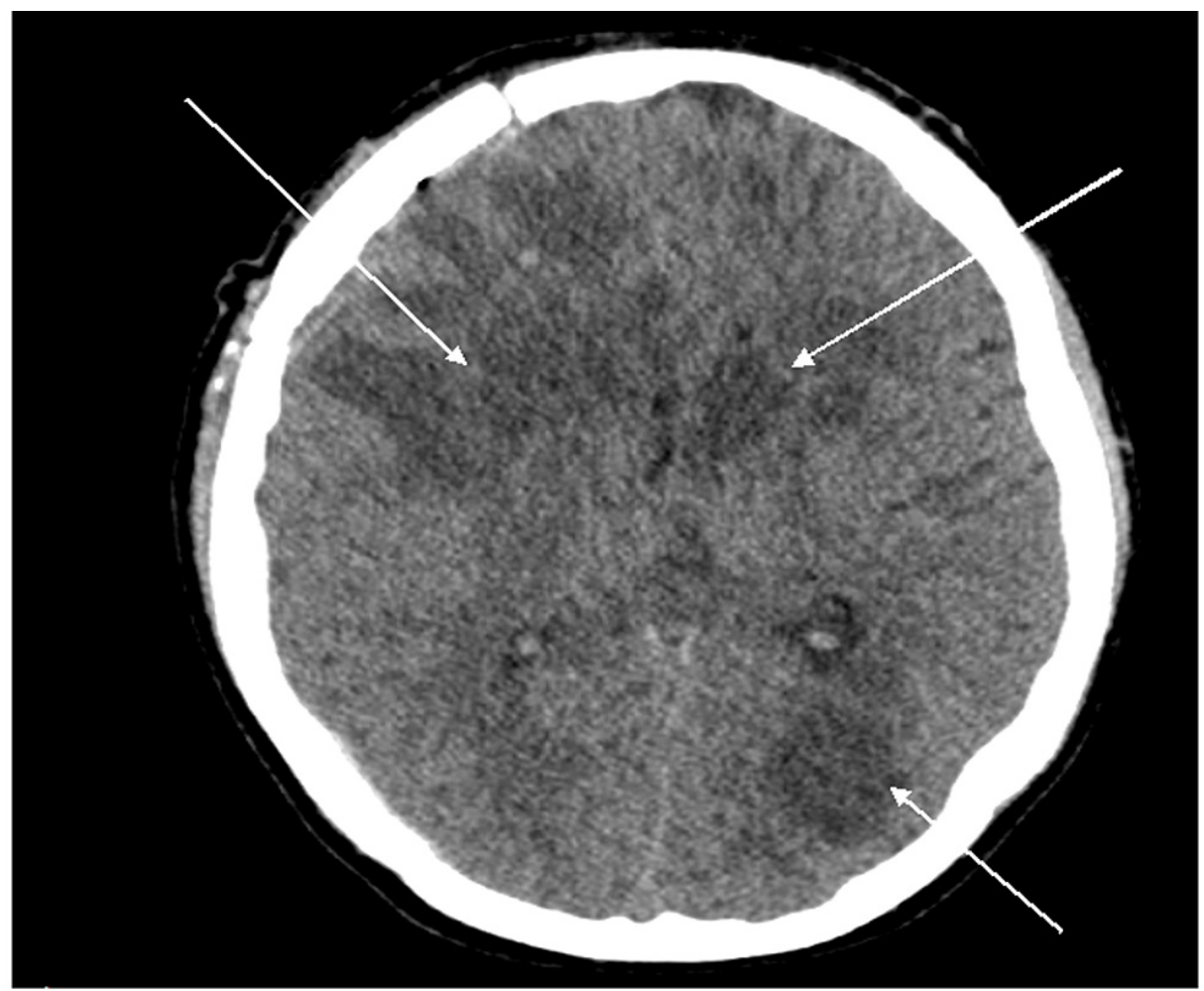

FIGURE 3: Post-craniotomy computed tomography scan of the brain showing partial evacuation of the right frontal lobe lesion and multifocal hypodense lesions as shown by arrows in both cerebral hemispheres

After a month of hospitalization, the patient was discharged home. Oral voriconazole and intravenous amphotericin B were continued for the next two months. The patient could not be followed up in our outpatient department because of affordability issues. Therefore, he continued medications under the care of a public sector hospital. We remained in contact with the patient via telephonic communication. After completing the three-month antifungal course, the patient was re-evaluated. Analysis of the aspirate from the persistent right-sided lesion revealed the recurrence of Acremonium species. The patient was started again on intravenous amphotericin $\mathrm{B}$ and oral voriconazole for the next three months.

\section{Discussion}

The emergence of many fungi, which were once considered pathogenically insignificant, as new etiological agents of various clinical conditions poses a serious diagnostic and therapeutic challenge. The increased incidence of fungal infections is associated with significant morbidity and mortality [4]. Although Aspergillus and Fusarium are the two most pathogenic filamentous fungi, the involvement of Acremonium species is being increasingly recognized in both localized and systemic infections [3,5]. The genus Acremonium is highly polyphyletic and consists of hyaline filamentous molds [1].

In immunocompetent hosts, Acremonium species can cause cutaneous and subcutaneous infections, such as superficial skin infections, mycetoma, endophthalmitis, and keratitis, usually after trauma [6]. On the other hand, in immunocompromised individuals, these fungi mainly cause invasive infections, manifesting in the form of osteomyelitis, arthritis, pneumonia, peritonitis, or disseminated infections, which affect a multitude of organ systems [6].

We report the first case of brain abscess caused by Acremonium species in a patient with no identifiable comorbidities in Pakistan. Brain abscess is a serious and potentially life-threatening emergency that requires prompt diagnosis and treatment. Any delay in the management of brain abscesses can lead to poor health outcomes. Both pharmacological and surgical treatments may prove to be very significant in managing fungal brain abscesses [7,8]. However, given the rarity of Acremonium infection and the limited published literature available, there are no established guidelines regarding its treatment.

In vitro data have revealed that Acremonium species are resistant to many antifungal drugs $[1,6]$. Newer azoles, such as voriconazole and posaconazole, may be effectively used against this fungus $[9,10]$. However, flucytosine, echinocandins, and fluconazole are not active against it $[11,12]$. 
In previous studies, deep tissue infections, such as a right-sided pacemaker-related endocarditis and pneumonia, caused by Acremonium species have been successfully treated with voriconazole $[8,13]$. Mattei et al. reported the successful treatment of two cases of fungemia with voriconazole [12]. Similarly, Herbrecht et al. reported the successful treatment of a case of pulmonary fungal infection with posaconazole [14]. Both voriconazole and posaconazole have high efficacy, good oral bioavailability, and high concentration in tissues, including those of the central nervous system (CNS) [15]. However, the recurrence of fungi has been reported after treatment with voriconazole [16]. In the present case, the use of intravenous amphotericin B and voriconazole initially caused great improvement; however, the recurrence of Acremonium species was noted after three months of completion of the antifungal course.

\section{Conclusions}

Although Acremonium is normally considered to be present in the environment, it has the potential to cause serious infections in humans. In this case report, we highlight the importance of performing a thorough evaluation for the identification of uncommon infectious diseases. In addition, it is essential for infectious disease specialists, neurosurgeons, and microbiologists to follow a multidisciplinary approach for the effective delivery of care in cases of rare, life-threatening fungal infections involving the CNS. Furthermore, the long-term use of newer azoles along with amphotericin B in cases of invasive fungal infections may result in reduced morbidity.

\section{Additional Information \\ Disclosures}

Human subjects: Consent was obtained or waived by all participants in this study. Clinical Research Committee, Ziauddin University issued approval 2883320AJPHA. Consent was obtained from the participant involved in the present study. Conflicts of interest: In compliance with the ICMJE uniform disclosure form, all authors declare the following: Payment/services info: All authors have declared that no financial support was received from any organization for the submitted work. Financial relationships: All authors have declared that they have no financial relationships at present or within the previous three years with any organizations that might have an interest in the submitted work. Other relationships: All authors have declared that there are no other relationships or activities that could appear to have influenced the submitted work.

\section{References}

1. Perdomo H, Sutton DA, García D, et al.: Spectrum of clinically relevant Acremonium species in the United States. J Clin Microbiol. 2011, 49:243-56. 10.1128/JCM.00793-10

2. Das S, Saha R, Dar SA, Ramachandran VG: Acremonium species: a review of the etiological agents of emerging hyalohyphomycosis. Mycopathologia. 2010, 170:361-75. 10.1007/s11046-010-9334-1

3. Fincher RM, Fisher JF, Lovell RD, Newman CL, Espinel-Ingroff A, Shadomy HJ: Infection due to the fungus Acremonium (cephalosporium). Medicine (Baltimore). 1991, 70:398-409. 10.1097/00005792-19911100000005

4. Kant R, Kaur T, Gupte S, Kaur M: A review on emerging fungal infections and their significance . J Bacteriol Mycol. 2015, 1:39-41. 10.15406/jbmoa.2015.01.00009

5. Nucci M, Anaissie E: Emerging fungi. Infect Dis Clin North Am. 2006, 20:563-79. 10.1016/i.idc.2006.06.002

6. Guarro J, Gams W, Pujol I, Gené J: Acremonium species: new emerging fungal opportunists--in vitro antifungal susceptibilities and review. Clin Infect Dis. 1997, 25:1222-9. 10.1086/516098

7. Aljuboori Z, Hruska R, Yaseen A, Arnold F, Wojda B, Nauta H: Fungal brain abscess caused by "Black Mold" (Cladophialophora bantiana) - A case report of successful treatment with an emphasis on how fungal brain abscess may be different from bacterial brain abscess. Surg Neurol Int. 2017, 8:46. 10.4103/sni.sni 44816

8. Klimko NN, Khostelidi SN, Melekhina YE, Gornostaev DA, Semelev VN, Bogomolova TS, Tirenko VV: Acremonium pneumonia successfully treated in patient with acute myeloid leukemia: a case report . J Bacteriol Mycol. 2016, 2:116-9. 10.15406/jbmoa.2016.02.00036

9. Espinel-Ingroff A: Comparison of In vitro activities of the new triazole SCH56592 and the echinocandins MK-0991 (L-743,872) and LY303366 against opportunistic filamentous and dimorphic fungi and yeasts. J Clin Microbiol. 1998, 36:2950-6. 10.1128/TCM.36.10.2950-2956.1998

10. Fernández-Silva F, Capilla J, Mayayo E, Sutton DA, Hernández P, Guarro J: Evaluation of the efficacies of amphotericin B, posaconazole, voriconazole, and anidulafungin in a murine disseminated infection by the emerging opportunistic fungus Sarocladium (Acremonium) kiliense. Antimicrob Agents Chemother. 2013, 57:6265-9. 10.1128/AAC.01484-13

11. Tortorano AM, Richardson M, Roilides E, et al.: ESCMID and ECMM joint guidelines on diagnosis and management of hyalohyphomycosis: Fusarium spp., Scedosporium spp. and others. Clin Microbiol Infect. 2014, 20 Suppl 3:27-46. 10.1111/1469-0691.12465

12. Mattei D, Mordini N, Lo Nigro C, Gallamini A, Osenda M, Pugno F, Viscoli C: Successful treatment of Acremonium fungemia with voriconazole. Mycoses. 2003, 46:511-4. 10.1046/j.0933-7407.2003.00924.X

13. Heitmann L, Cometta A, Hurni M, Aebischer N, Tschan-Schild I, Bille J: Right-sided pacemaker-related endocarditis due to Acremonium species. Clin Infect Dis. 1997, 25:158-60. 10.1086/516892

14. Herbrecht R, Letscher-Bru V, Fohrer C, Campos F, Natarajan-Ame S, Zamfir A, Waller J: Acremonium strictum pulmonary infection in a leukemic patient successfully treated with posaconazole after failure of amphotericin B. Eur J Clin Microbiol Infect Dis. 2002, 21:814-7. 10.1007/s10096-002-0828-8

15. Greenberg RN, Mullane K, van Burik JA, et al.: Posaconazole as salvage therapy for zygomycosis. Antimicrob 


\section{Cureus}

Agents Chemother. 2006, 50:126-33. 10.1128/AAC.50.1.126-133.2006

16. Tuon FF, Pozzi C, Penteado-Filho SR, Benvenutti R, Contieri FL: Recurrent Acremonium infection in a kidney transplant patient treated with voriconazole: a case report. Rev Soc Bras Med Trop. 2010, 43:467-8. 10.1590/s0037-86822010000400028 